\title{
DERIVATION OF STRONG INTERACTIONS FROM A QUARK MODEL*
}

\author{
PETER G.0. FREUND and ENRICO PREDAZZI ${ }^{\dagger}$ \\ The Enrico Fermi Institute for Nuclear Studies \\ and the Department of Physics \\ The University of Chicago, Chicago, Illinois \\ (Received 6 May 1966)
}

\begin{abstract}
A model of mesons as quark-antiquark bound states is presented. The model is based on a zero range approximation and SU(3)-symmetry. It yields the following results: (1) The couplings of the mesons to quarks can be calculated and they conform to the same pattern that would be followed by couplings among multiplets of the group $U(6) \times U(6) \times O(3)$. Thus $U(6) \times U(6) \times O(3)$ symmetry emerges as a consequence of the dynamical features of our model. (2) Beyond the high symmetry that it produces, our model predicts the numerical value of the universal strong coupling constant in terms of the ratio of meson to quark masses. This fact when used in conjunction with the experimentally known value of the universal strong coupling constant leads to a very low value for the quark mass $M$, namely $M \approx 1 / 2 \times$ (central meson mass). (3) Using this value for the quark mass, the $\pi \rightarrow \mu \nu$ decay rate is estimated from the quark model in much the same way as one calculates the $2 \gamma$ decay rate of parapositronium. The result agrees remarkably well with experiment. (4) It is argued that the possible existence of quarks with a mass of the order of half a $\mathrm{BeV}$ may not be excluded by existing experiments.
\end{abstract}

\section{Introduction}

THE broken $S U(6)$-symmetry and approximate universality of strong interactions can be interpreted as consequences of a quark $(q)$ substructure of hadrons. The experimental situation concerning the actual existence of quarks is presently not clear. In spite of this fact, we shall present here a theory of mesons of spin parities $0^{ \pm}, 1^{ \pm}$as $\bar{q} q$ bound states. Our theory is relativistic and satisfies the condition of unitarity for the $S$-matrix. It is based on a zero-range approximation first proposed in its full generality by Landau [1]. The meson-quark couplings can then be calculated in terms of the meson to quark mass ratios (Section 2).

As a consequence of the dynamics of our model, these couplings turn out to follow the same

This work supported in part by the U.S. Atomic Energy Commission, Contract No. AT(11-1)264.

$\dagger$ Enrico Fermi Fellow. On leave-of-absence from the Institute of Physics of the University of Torino, Italy. 
pattern that would characterize the couplings of multiplets of the group $U(6) \times U(6) \times O(3)$, (Sections 3 and 4). Thus $S U(3)$-symmetry, which we assume as an input in the context of zero range dynamics, creates the large $U(6) \times U(6) \times O(3)$ symmetry. Since we deal with an explicit dynamical scheme, we are able to make quantitative predictions even on the strength of the strong interactions. Such predictions are beyond the reach of any type of symmetry considerations. From the experimental value of the universal strong interactions, we are able to calculate the quark mass $M$ and obtain the surprisingly low value $M \approx 1 / 2 \times$ central meson mass (Section 5). With this value for $M$, we then estimate the $\pi \rightarrow \mu \nu$ decay rate using the method familiar from the calculation of the $2 \gamma$ annihilation rate of parapositronium (Section 6). The result is in good agreement with experiment. We then discuss possible barrier effects that woul contain quarks in hadrons and thus account for the lack of copious quark production at experimental energies (Section 7). The implications of our work are discussed in Section 8.

\section{The Zero Range Bound State Model}

In this section we shall treat mesons as $q \bar{q}$ bound states. Our treatment will be based on a zero-range approximation [1]. Specifically, we shall construct relativistic zero-range expressions for the $q \bar{q}$ partial wave amplitudes. These expressions are then normalized by imposing elastic unitarity near the $q \bar{q}$ threshold. The so normalized partial waves are then analytically continued to the position of the bound-state pole and the corresponding residuum is then obtained in terms of the ratio $\alpha=2 M / m, M$ being the quark mass and $m$ the mass of the bound state In this way, we are able to calculate the coupling constants of quarks to $0^{ \pm}, 1^{ \pm}, \ldots$ mesons. We are, of course, not in measure to calculate the position of the bound state pole, as we do not make any specific assumptions about the inter-quark interaction except for its zero range.

Before going into details, we would still like-to clarify the field-theoretic meaning of our approximation. If quarks were scalar particles then, as has been shown by Thirring [2], this approach is essentially equivalent to summing $q \bar{q}$-bubble graphs. Each of the bubbles would then be logarithmically divergent so that one would not be able to determine the position of the pole. However, given the position of the pole, one is allowed an extra subtraction so that the coupling constant (residuum) converges and for small binding energies is identical to the zerorange approximation. Even for large binding energies $(\alpha \rightarrow \infty)$, the two models agree to within a factor two or so. This then indicates that the zero-range approximation may be valid also beyond the region of small binding energies.

We shall treat a more realistic case in which quarks are endowed with spin and unitary spin. In this case we still obtain finite values for the coupling constants, but the straightforward equivalence of our model and the bubble diagrams is lost. The bubble diagrams are now quadratically divergent and their sum still leaves a logarithmic divergence in the expression for the coupling constant. This presumably means that in a field-theoretic approach we would have to use a cutoff. Independently of its field theoretic meaning, let us emphasize that for small binding energies $(\alpha \rightarrow 1)$ the zero-range approximation is a very good approximation. We shall see that in the range of small binding energies a very interesting solution for the meson states emerges.

Now let us go into the details of the $q \bar{q}$ problem. The quarks are as usual an $S U(3)$-triplet of spin $1 / 2$ particles. $q \bar{q}$ scattering can correspondingly be described in terms of five $S U(3)$ singlet and five $S U(3)$-octet invariant amplitudes. For both $S U(3)$-singlet and octet we shall choose these five amplitudes to be the five helicity amplitudes $\phi_{1}, \ldots, \phi_{5}$ defined by Goldberger et al. [3]. In terms of the $\phi^{\prime} s$ we define following reference [3] partial wave projections 
${ }_{0}^{X J}, h_{1}^{X J}, h_{l=J-1}^{X J}, h_{l=J+1}^{X J}$ and $h_{J-1, J+1}^{X J}$ with $X=1$ and 8. These amplitudes correspond espectively to the transitions ${ }^{1} J_{J} \rightarrow{ }^{1} J_{J},{ }^{3} J_{J} \rightarrow{ }^{3} J_{J},{ }^{3}(J-1)_{J} \rightarrow{ }^{3}(J-1) J$, $(J+1)_{J} \rightarrow{ }^{3}(J+1)_{J}$ and ${ }^{3}(J-1)_{J} \longleftrightarrow^{3}(J+1)_{J}$. Furthermore, our $h$ 's are normalzed differently from the corresponding $f^{\prime} \mathrm{s}$ of Goldberger et al. [3]. To wit $h^{X J}=(1 / p) f^{X J}$, here $p$ is the $q \bar{q}$ c.m. momentum [4]. We now introduce the matrices

$$
H^{x J}=\left(\begin{array}{cccc}
n_{0}^{x J} & 0 & 0 & 0 \\
0 & n_{1}^{x J} & 0 & 0 \\
0 & 0 & n_{l=j-1}^{x J} & n_{j-1, J+1}^{x J} \\
0 & 0 & n_{j=1, J+1}^{x J} & n_{l=j+1}^{x J}
\end{array}\right)_{;} x=1,8 .
$$

The condition of elastic unitarity for the $q \bar{q}$ partial waves can be stated in the form of the atrix equations

$$
\operatorname{Im} H^{X J}=p\left(H^{X J}\right)+H^{X J} \text {. }
$$

The zero range approximation then amounts to choosing the explicit solution $H^{X J}$ of equation (2) given by

$$
H^{X J}=\left(\begin{array}{ccc}
\frac{-\xi_{0}^{x J}}{a_{0}^{x J}+i p} & 0 \\
0 & \frac{-\xi_{1}^{x J}}{a_{1}^{x J+i p}} & 0 \\
& 0 & A^{x J}
\end{array}\right) \text {, }
$$

$$
A^{x \omega}=-\frac{1}{a_{2}^{x J}+i p}\left(\begin{array}{ccc}
\xi_{2}^{x J} & \left(\xi_{2}^{X J}\right. & \left.\xi_{3}^{X J}\right)^{1 / 2} \\
\left(\xi_{2}^{x J}\right. & \left.\xi_{3}^{X J}\right)^{N 2} & \xi_{3}^{X J}
\end{array}\right)
$$

and the $\xi$ s obey

$$
\xi_{0}^{X J}=1, \quad \xi_{1}^{X J}=1, \quad \xi_{2}^{X J}+\xi_{3}^{X J}=1 .
$$


If there is a bound state in a given partial wave, then its position will be

$$
\left(m_{z}^{X J}\right)^{2}=4\left[M^{2}-\left(a_{z}^{X J}\right)^{2}\right], \quad(z=0,1,2) .
$$

If, on the other hand, there is no bound state, this can be expressed in our picture by letti the corresponding $a \rightarrow \infty$.

We now have to interpret the solutions (3-6) in terms of bound state particles of given sp parity and charge conjugation quantum numbers. To do this, we write down the most general cou ling of quarks to mesons with $J^{P C}=0^{-+}, 1^{--}, 0^{++}, 1^{++}$and $1^{+-}$. Let us call the respective mesons $P^{X}, V_{\mu}^{X}, S^{X}, A_{\mu}^{X}$ and $B_{\mu}^{X}$, and the corresponding masses $m_{P}^{X}, m_{V}^{X}, m_{S}^{X}, m_{A}^{X}$ and $m_{B}^{X}$. The couplin are:

$$
\begin{aligned}
& \Gamma=\sqrt{2} \bar{w}(\bar{p})\left\{\left(g_{p}^{8} \gamma_{5} p^{8}+g_{p}^{1} \gamma_{5} p^{1}\right)\right. \\
& +g_{V}^{8}\left(\gamma^{\mu}+\eta_{8} \frac{K^{\mu}}{2 m}\right) v_{\mu}^{8}+g_{V}^{1}\left(\gamma^{\mu}+\eta_{1} \frac{K^{\mu}}{2 \mu}\right) v_{\mu}^{1} \\
& +\left(g_{S}^{8} s^{8}+g_{S}^{1} s^{1}\right) \\
& +\left(g_{A}^{8} ; \gamma^{\mu} \gamma_{5} A_{\mu}^{8}+g_{A}^{1} ; \gamma^{\mu} \gamma_{5} A_{\mu}^{1}\right) \\
& \left.+\left(g_{B}^{8} \kappa^{\mu} \gamma_{5} B_{\mu}^{8}+g_{B}^{1} \kappa^{\mu} \gamma_{5} \delta_{\mu}^{\prime}\right)\right\} u(p) .
\end{aligned}
$$

Here

$$
\begin{aligned}
& w(\bar{p})=\sqrt{\frac{E-M}{2 M}}\left(\frac{\sigma^{\phi} \bar{p}}{E-M} \phi\right), \mu(p)=\sqrt{\frac{E+M}{2 M}}\left(\frac{\sigma^{\circ} p}{E+M} x\right) \\
& \bar{w}(\bar{p})=w^{+}(\bar{p}) \gamma_{0}, K^{\mu}=(p-\bar{p})^{\mu} ;
\end{aligned}
$$

and we use the metric $(1,-1,-1,-1)$, the $\gamma$ matrices $\gamma_{0}=\left({ }^{1}-1\right), \gamma=\left(\sigma_{-} 9\right), \gamma_{5}=\left({ }_{1}^{1}\right)$. Also, as is obvious from equation (8) $(\gamma p-M) u(p)=(\gamma \bar{p}+M) w(\bar{p})=0$. Furthermore

$$
\rho^{8}=\left(\begin{array}{ccc}
\frac{\eta}{\sqrt{6}}+\frac{\pi^{0}}{\sqrt{2}} & \pi^{+} & \kappa^{+} \\
\pi^{-} & \frac{\eta}{\sqrt{6}}-\frac{\pi^{0}}{\sqrt{2}} & \kappa^{0} \\
\kappa^{-} & k^{0} & \frac{-2 \eta}{\sqrt{6}}
\end{array}\right) ; \rho^{\prime}=\left(\begin{array}{ccc}
\frac{x^{0}}{\sqrt{3}} & 0 & 0 \\
0 & \frac{x^{0}}{\sqrt{3}} & 0 \\
0 & 0 & \frac{x^{0}}{\sqrt{3}}
\end{array}\right)
$$


being the ninth $0^{-}$meson and a similar representation for the vector meson matrices $V_{8}$ and $V_{1}$ $B$ used (because of the assumption of exact $S U(3)$ symmetry $\omega-\phi$ mixing can be ignored).

We can now calculate the $\xi$ 's appearing in the matrix $H$ (equations (3) and (4)) in terms of he 12 coupling constants ( $g^{\prime} s$ and $\eta^{\prime} s$ ) appearing in equation ( 7$)$. The result is:

$$
\begin{aligned}
& \xi_{0}^{x O}=\frac{1}{2} c_{p x}^{(-)} \\
& \xi_{2}^{x 1}=\frac{2}{9} c_{v x}^{(-)}\left\{1+\frac{a_{v x}}{2}\left(1+\eta_{x}-\frac{\eta_{x}}{a_{v x}^{2}}\right)\right\}^{2} \\
& \xi_{3}^{x 1}=\frac{1}{9} c_{v x}^{(-)}\left\{1-a_{v x}\left(1+\eta_{x}-\frac{\eta_{x}}{a_{v x}^{2}}\right)\right\}^{2} \\
& \xi_{3}^{x 0}=\frac{1}{2} c_{s x}^{(+)} \\
& \xi_{1}^{x 1}=\frac{1}{3} c_{A x}^{(+)} \\
& \xi_{0}^{x 1}=\frac{1}{6 a_{B X}^{2}} c_{B X}^{(+)}
\end{aligned}
$$

there

$$
\begin{aligned}
& C_{i X}^{( \pm)}=\frac{g_{i X}^{2}}{4 \pi}\left(\alpha_{i X}^{2}-1\right)^{ \pm / / 2} \\
& \alpha_{i X}=2 M / m_{i X}
\end{aligned} \quad\left(\begin{array}{l}
i=P, V, S, A, B \\
X=1,8
\end{array}\right)
$$

and the $g_{i X}{ }^{\prime} s$ are those defined in (7). Observe that the poles in the ${ }^{1} S_{0},{ }^{3} S_{1},{ }^{1} P_{1},{ }^{3} P_{0},{ }^{3} P_{1}$ partial wave amplitudes are due to the $P, V, A, S, B$ mesons as expected.

Imposing the unitarity conditions (5), we find from equations (10) and (11):

$$
\begin{aligned}
& g_{p x}^{3} / 4 \pi=2\left(a_{p x}^{2}-1\right)^{1 / 2} \\
& g_{v x}^{2} / 4 \pi=3\left(a_{v x}^{2}-1\right)^{1 / 2}\left[1+\frac{a_{v x}^{2}}{2}\left(1+\eta_{x}-\frac{\eta_{x}}{a_{v x}^{2}}\right)^{2}\right]^{-1} \\
& g_{s x / 4 \pi}^{2}=2\left(a_{s x}^{2}-1\right)^{-1 / 2}
\end{aligned}
$$




$$
\begin{aligned}
& g_{A X}^{2} / 4 \pi=3\left(a_{A X}^{2}-1\right)^{-1 / 2} \\
& g_{B X}^{2} / 4 \pi=6 a_{B X}^{2}\left(a_{B X}^{2}-1\right)^{-1 / 2}
\end{aligned}
$$

where $\alpha_{i X}$ and $\eta_{X}$ are defined by equations (11) and (7) respectively.

Equations (12) are the central result of this section. We observe first that in the limit of small binding energies all $P$ and $V$ coupling constants become proportional to the square root of the binding energy $\sqrt{B}$. This is in keeping with the interpretation of the $P$ and $V$ mesons as $q \bar{q}-S$ states [1]. On the other hand, for $S, A$ and $B$ mesons we find in this limit $g^{2} / 4 \pi \sim 1 / \sqrt{B}$ as befits $P$-states. It is important to remark that equations (12) determine all $\mathrm{g}$ ' $s$ appearing in (7) in terms of the mass ratios $\alpha_{i X}$ and of the $P_{\mu}$ to $\gamma_{\mu}$ type vector meson coupling ratios $\eta_{1}$ and $\eta_{8}$. However, equations (12) do not determine $\eta_{1}$ and $\eta_{8}$.

\section{Predictions of $U(6) \times U(6) \times 0(3)$ Symmetry for Meson Quark Couplings}

The quantum numbers of the quarks and mesons we treated in Section 2 are such that they could be classified in multiplets according to the group $U(6) \times U(6) \times O(3)$. In this section we wish to establish the predictions of this symmetry scheme for quark-meson couplings. Our reason for doing this is that in the next section we are going to compare the predictions of $U(6) \times U(6) \times O(3)$ symmetry with the predictions of the zero range approximation presented in Section 2. We shall see in the next section that the two schemes are equivalent.

Now to get started, we shall assume that quarks belong to the representation $(6,1, L=0)$, and antiquarks to $(1, \overline{6}, L=0)$. The $0^{-}(P)$ and $1^{-}(V)$ mesons appear in $(6, \overline{6}, L=0)$; the $0^{+}(S)$, $1^{+}(A), 1^{+}(B)$ and the $2^{+}(T)$ mesons in $(6, \overline{6}, L=1)$. This classification, as is well known, implies

$$
m_{P_{8}}=m_{P_{1}}=m_{V 8}=m_{V 1}=m_{0}
$$

and

$$
m_{S 8}=m_{S 1}=m_{A 8}=m_{A 1}=m_{B 8}=m_{B 1}=m_{T 8}=m_{T 1}=m_{1}
$$

We can now describe the $L=0$ mesons by the usual matrix

$$
\mathscr{M}(q)=\left(1+\frac{\gamma q}{m_{0}}\right)\left(\gamma_{5} P+\gamma_{\mu} V^{\mu}\right)
$$

where

$$
P=P^{8}+P^{1}, \quad V_{\mu}=V_{\mu}^{8}+V_{\mu}^{1}
$$

and $P^{8}, \ldots, V_{\mu}^{1}$ are the matrices defined by equations (9). Similarly for the $L=1$ mesons we can use the kinetic supermultiplet matrix [5] 


$$
\begin{aligned}
& \mathscr{R}_{\nu}(q)=\left(1+\frac{\gamma q}{m_{1}}\right)\left[\gamma ^ { \mu } \left\{T_{\mu \nu}+\varepsilon_{\mu \nu \rho \sigma} \frac{q^{\rho}}{m_{1}} A^{\sigma}\right.\right. \\
& \left.\left.+\sqrt{\frac{2}{3}}\left(g_{\mu \nu}-\frac{q_{\mu} q_{\nu}}{m_{1}^{2}}\right) S\right\}+\sqrt{2} \gamma_{5} B_{\nu}\right] .
\end{aligned}
$$

here the mesons in the parenthesis have the same meanings as in the previous sections $\left(T_{\mu \nu}=\right.$ $v_{\mu \mu}$ is the $2^{+}$meson) and, of course, obey the subsidiary conditions $q^{\mu} T_{\mu \nu}=q^{\mu} A_{\mu}=q^{\mu B_{\mu}}=0$. me couplings of $\mathscr{M}$ and $\mathscr{R}_{\mu}$ to quarks are given by [6]

$$
\begin{aligned}
& \Gamma_{u(6) \times u(6) \times o(3)}=\frac{\sqrt{2}}{1+\alpha_{0}}\left[g_{0} \bar{w}(\bar{p}) M(q) u(p)\right. \\
& \left.+\frac{1}{12} g^{\prime} \bar{w}(\bar{p}) u(p) \pi\left(\frac{\gamma K}{m} M(q)\right)\right] \\
& +\frac{\sqrt{2}}{1+a_{1}}\left[g_{1} \bar{w}(p) K^{\mu} R_{\mu}(q) \cup(p)\right. \\
& \left.+\frac{1}{12} g_{1}^{e} \bar{\nabla}(\bar{p}) \cup(p) \pi\left(\frac{Y K}{m} K^{\mu} R_{\mu}(q)\right)\right],
\end{aligned}
$$

where $\alpha_{0}=2 M / m_{0}, \alpha_{1}=2 M / m_{1},-q=p+\bar{p}$. In equation (18) the unprimed terms correspond to what may well be called "exact" $M(12) \times O(3)$ symmetry [6] whereas inclusion of the primed (kineton) terms corresponds to restricting ourselves to the collinear group $[S U(6) \times O(2)]_{\text {collinear. In }}$ case we interpret this symmetry breakdown as a kinetic energy effect, we should expect [6]

$$
\begin{aligned}
& g_{0}^{\prime} \ll g_{0} . \\
& g_{1}^{\prime} \ll g_{1} .
\end{aligned}
$$

Now in terms of the six parameters $g_{0}, g_{1}, g_{0}^{\prime}, g_{1}^{\prime}, \alpha_{0}=2 M / m_{0}$ and $\alpha_{1}=2 M / m_{1}$, the coupling constants in the normalization specified by equation (7) are

$$
\begin{aligned}
& \frac{g_{p_{8}}^{2}}{4 \pi}=\frac{g_{P_{1}}^{2}}{4 \pi}=\frac{g^{2}}{4 \pi}=\frac{g_{1}^{2}}{4 \pi}=\frac{g_{0}^{2}}{4 \pi} \\
& \eta_{8}=\eta_{1}\left(1+g_{0}^{\prime} / g_{0}\right)=-\frac{a_{0}}{1+\alpha_{0}}
\end{aligned}
$$




$$
\begin{aligned}
& \frac{3}{2} \frac{g_{s 8}^{2}}{4 \pi}=\frac{g_{48}^{2}}{4 \pi}=\frac{1}{2 \alpha_{1}^{2}} \frac{g_{B 8}^{2}}{4 \pi}=\frac{g_{A 1}^{2}}{4 \pi}=\frac{1}{2 \alpha_{1}^{2}} \frac{g_{B 1}^{2}}{4 \pi}=\frac{g_{1}^{2}}{4 \pi} \\
& \frac{3}{2} \frac{g_{s 1}^{2}}{4 \pi}=\frac{\left[g_{1}+\left(a_{1}-1\right) g_{1}^{\prime}\right]^{2}}{4 \pi} .
\end{aligned}
$$

In the next section we shall compare these predictions with the predictions of our zero-range approximation (Section 2).

Before concluding this section, we wish to reemphasize that our dynamical (Section 2) and $U(6) \times U(6) \times O(3)$ symmetry (this section) considerations are completely independent. Nowhere in obtaining equations (12) have we used any $U(6) \times U(6) \times O(3)$ arguments and, on the other hand, the equations $(20)$ are independent of dynamics.

\section{Derivation of $U(6) \times U(6) \times 0(3)$ Symetry Results from Zero-Range Approximation}

In Section 2 we used the dynamics of a zero-range approximation to express the coupling con stants of $0^{ \pm}$and $1^{ \pm}$mesons to quarks in terms of the meson masses and of the quark mass. We were, of course, not able (as explained in Section 2) to calculate the masses of the mesons. Now let us assume that the $L=0$ mesons $V$ and $P$ have comparable masses $m_{V} \approx m_{P} \approx m_{0}$ and that the $L=1$ mesons $S, A, B$ and $T$ have also comparable masses $m_{S} \approx m_{A} \approx m_{B} \approx m_{T} \approx m_{1}$. In other words, we supplement our dynamical relations (12) by the equations (13) and (14). We are now in measure to calculate the ratios of the various coupling constants and find:

$$
\begin{aligned}
& g_{P \delta}^{2} / 4 \pi: g_{P 1}^{2} / 4 \pi: g_{V 8}^{2} / 4 \pi: g_{V 1}^{2} / 4 \pi \\
& =1: 1: \frac{3}{2}\left[1+\frac{a_{0}^{2}}{2}\left(1+\eta_{8}-\frac{\eta_{R}}{a_{\delta}^{2}}\right)^{2}\right]^{-1}: \frac{3}{2}\left[1+\frac{a_{0}^{2}}{2}\left(1+\eta_{1}-\frac{\eta_{1}}{a_{f}^{2}}\right)^{2}\right]^{-1}, \\
& g_{S 8}^{2} / 4 \pi: g_{S 1}^{2} / 4 \pi: g_{A 8}^{2} / 4 \pi: g_{A 1}^{2} / 4 \pi: g_{B 8}^{2} / 4 \pi: g_{B 1}^{2} / 4 \pi \\
& =1: 1: \frac{3}{2}: \frac{3}{2}: 3 a_{1}^{2}: 3 a_{1}^{2} .
\end{aligned}
$$

where we again used the notations $\alpha_{0}=2 M / m_{0}, \alpha_{1}=2 M / m_{1}$. On the other hand from our $U(6) x$ $U(6) \times O(3)$ symmetry considerations of Section 3 (equation 20), we can also calculate ratios of coupling constants, and we find

$$
\begin{aligned}
& g_{s 8}^{2} / 4 \pi: g_{p 1}^{2} / 4 \pi: g_{8}^{2} / 4 \pi: g_{n}^{2} / 4 \pi=1 \\
& g_{s 8}^{2} / 4 \pi: g_{s 1}^{2} / 4 \pi: g_{s_{0}}^{2} / 4 \pi: g_{11}^{2} / 4 \pi: g_{s}^{2} / 4 \pi: g_{01}^{2} / 4 \pi \\
& =1:\left[1+\left(a_{1}-1\right) \frac{g_{1}^{0}}{g_{1}}\right]^{2}: \frac{3}{2}: \frac{3}{2}: 3 a_{1}^{2}: 3 a_{1}^{2},
\end{aligned}
$$




$$
\eta_{8}=\eta_{1}\left(1+\frac{g_{0}^{\prime}}{g_{0}}\right)^{-1}=-\frac{\alpha_{0}}{1+\alpha_{0}} .
$$

Now observe that the ratios (21) depend on two parameters $\eta_{1}$ and $\eta_{8}$, whereas the ratios (22) epend on the two parameters $g_{0}^{\prime} / g_{0}$ and $g_{i}^{\prime} / g_{1}$. All ratios that are independent of these paraeters in the two sets (and these actually constitute the majority) coincide. Furthermare, the arameter-dependent ratios are compatible and can be used to determine the values of the paraeters. Indeed, using the values of $\eta_{8}$ and $\eta_{1}$ given by equation (22b) we find from equations 21) and (22a) [6]

$$
g_{0}^{\prime}=g_{i}^{\prime}=0 .
$$

hus we find that our dynamical model completely reproduces $U(6) \times U(6) \times O(3)$ symmetry and oreover implements conditions (19) thus achieving what we call "exact" $M(12) \times O(3)$ symmetry. his is a highly remarkable result since, as is obvious from Section 2, we have nowhere in our odel assumed anything more than $S U(3)$ symmetry. The large output symmetry is a consequence of ero range dynamics in our scheme. Remark that the agreement between (21) and (22) is independnt of the values of $\alpha_{0}$ and $\alpha_{1}$. We have not discussed above the spin $2^{+}$mesons for the $P$-wave $\bar{q}$ states have only been included into our discussion for purposes of illustrating the generalty of our results. The basic interest is, of course, focused on the $P$ and $V$ mesons (see also ext section). It should also be pointed out that our treatment could be easily generalized to he case when some of the particles were $q \bar{q}$ resonances rather than bound states. This possiility is interesting in view of the results (29a) and (30a) of the next section but will not e considered here. We just wish to mention the amusing case when some even higher degeneracy ppears, namely that between $S$ - and $P-q \bar{q}$ states. In the notations introduced above this means $z_{0}=m_{1}$ or alternatively $\alpha_{0}=\alpha_{1}$. In this case we find that the products (not ratios) of the pouplings to quarks of one odd-parity meson and one-even parity meson are normalized. Thus we find from (12) using (13) and $m_{0}=m_{1}$ relations of the type

$$
\begin{aligned}
& g_{P 8 / 4 \pi}^{2} \cdot g_{A 8 / 4 \pi}^{2}=6 \\
& g_{P 8 / 4 \pi}^{2} \cdot g_{S 8 / 4 \pi}^{2}=4 .
\end{aligned}
$$

It is hard to understand how a higher symmetry (like e.g. $U(6) \times U(6) \times O(4)$ ) which could account for the degeneracy $m_{0}=m_{1}$, could also reproduce the relations (24).

Let us still emphasize that even in the presence of mass splittings among the mesons of various spins equations (12) can be used to determine the coupling constant pattern. This parallels the inclusion of $S U(6)$-breaking couplings at the group-theoretical level. Equations (12) actually reach beyond symmetry in their implications. They actually normalize the coupling constant in terms of the meson to quark mass ratios. It is this feature of equations (12) that carries the most valuable information which is typical of the explicit dynamical input and unobtainable by symmetry considerations of any kind.

\section{Determination of Quark Mass from Zero-Range Approximation}

As we have already pointed out in the last section our zero range bound state dynamics allow us to express the meson-quark coupling constants in terms of the meson to quark mass ratios. In 
fact equations (12) provide the necessary expressions.

In particular the first equation (12) gives

$$
\frac{g_{P 8}^{2}}{4 \pi}=2\left(\alpha_{0}^{2}-1\right)^{x} \text {. }
$$

From equations (25) and (7) we then find

$$
\frac{\mathrm{g}_{\pi^{0}}^{2} Q}{4 \pi}=2\left(\alpha_{0}^{2}-1\right)^{\mathrm{K}} \text {. }
$$

It has been shown on the basis of the universality of strong interactions that [7]

$$
\frac{g_{\pi^{0} Q}^{2}}{4 \pi}=\left(\frac{3}{5} \frac{M}{m_{\text {Baryon }}}\right)^{2} \frac{g_{\pi^{0} P}^{2}}{4 \pi},
$$

whence using [6] $m_{\text {Baryon }}=3 / 2 m$

$$
\frac{g_{\pi}^{2} Q}{4 \pi}=\frac{1}{25} \alpha_{0}^{2} \frac{g_{\pi}^{2} P}{4 \pi} .
$$

In equation (28), $\mathrm{g}_{\pi^{0} P / 4 \pi}^{2} \approx 15$. tions

From (26) and (28) we now find a second order algebraic equation for $\alpha_{0}^{2}$ which has the solu-

$$
\alpha_{0}=1.05 \text {, }
$$

or

$$
\alpha_{0}=3.16 \text {. }
$$

Using $m_{0} \approx 750 \mathrm{MeV}$ this means for the quark mass $M$

$$
M \approx 380 \mathrm{MeV},
$$

or

$$
M \approx 1180 \mathrm{MeV} \text {. }
$$

Both these values are strikingly low, In fact, of the two, (30a) offers the very amusing possibility of interpreting mesons as very loosely bound $q \bar{q}$-states [8]. We wish to emphasize that solution (30a) has the theoretical advantage of self-consistency. We mean by this that for the solution (30a), $\alpha_{0}$ being very close to unity, the zero range approximation is a very good one. of course the exact numerical values of equations (30) are not to be taken seriously for, e.g. $S U$ (3) mass-splittings are not included. What is meant by equation (30a) is that the quark masses are typically 400-600 MeV. In the following sections we will present further implications of the solution $(30 a)$. 


\section{Calculation of the $\pi \rightarrow \mu v_{\mu}$ Decay Rate in the Quark Model of Mesons}

It is well known that in a quark model of mesons the amplitude for the decay $\pi \rightarrow \mu \nu$ can be falculated [9]. In our model we shall use solution $(30 \mathrm{a})$ for the quark mass [10]. We can then isualize the meson as a loosely bound $q \bar{q}$-system in which the quarks have very small imaginary omenta. We can therefore proceed with the calculation of the $\pi \rightarrow \mu \nu$ decay rate much in the anner used in calculating the $2 \gamma$ decay rate of parapositronium [11]. In other words we shall xpress the matrix element $f_{\pi}$ for the process

$$
\pi \rightarrow \mu+\bar{v}_{\mu} .
$$

terms of the axial vector current contribution to the process

$$
(q \bar{q})_{3} s_{0}, t_{\text {rest }} \rightarrow \mu+\bar{v}_{\mu} .
$$

e define $f_{\pi}$ by the relation

$$
<0\left|j_{\mu}^{5}\right|_{\pi}>=\frac{1}{2} q_{\mu} f_{\pi} .
$$

$q_{\mu}=$ pion four momentum, $j_{\mu}^{5}$ weak axial vector current). The decay rate for process (31) is hen

$$
\frac{1}{\tau_{\pi}}=G^{2} \cos ^{2} \theta m_{\pi} m_{\mu}^{2}\left(1-\frac{m_{\mu}^{2}}{m_{\pi}^{2}}\right)^{2} \frac{f_{\pi}^{2}}{16 \pi}
$$

ith $G \approx 1.0110^{-5} m_{p}^{-2}, \theta \approx 0.26$. In terms of our quark model (32), then the relevant matrix lement is [12] $\left.(G / \sqrt{2}) \Psi(0) \bar{v}(\bar{p}) \gamma_{\mu \gamma} u(p)\right|_{p=\bar{p}=0}$ so that

$$
\left(\frac{f_{\pi}}{2}\right)^{2} \cdot=\frac{1}{m}|\Psi(0)|^{2} \text {. }
$$

There $|\Psi(0)|$ is the value of the quark wave function at the origin within the meson. of course $\mathbf{P}(0)$ would be exactly determined by solving say a Bethe-Salpeter equation. For our purposes we bhall assume

$$
|\Psi(0)|^{2} \approx\left(\pi r \frac{3}{\pi}\right)^{-1}
$$

where $r_{\pi}$ is the electric m.s. radius of the pion. Of course the exact value of the numerical coefficient could depart slightly from $\pi$ (e.g. $4 / 3 \pi$ etc. ...) but we do not expect this to appreciably affect our result. Furthermore in deriving equation (35) we have of course used a ifour Fermi interaction for process (32) which has the strength $(G / \sqrt{2})\left(G=1.0110^{-5} \mathrm{~m}_{P}^{-2}\right)[12]$. From equations (35) and (36) we find

$$
f_{\pi}=2\left(m \pi r_{\pi}^{3}\right)^{-1 / 2} \text {. }
$$

In the context of $S U(6)$ symmetry [13]

$$
\frac{r_{\pi}^{2}}{6}=\frac{r_{P}^{2}}{6}=\frac{1}{m^{2}}
$$


and equation (37) can be rewritten as

$$
J \pi=\frac{2 m}{(6 \pi \sqrt{6})^{1 / 2}} \approx 0.3 \mathrm{~m} .
$$

Equations (37) and (39) are the main results of this section. Inserting $m \approx 750 \mathrm{MeV}$, equation (39) gives

$$
\left(f_{\pi}\right)_{\text {theor. }} \approx 220 \mathrm{MeV} \text {. }
$$

From the experimental value $\tau_{\pi}=2.5510^{-8} \mathrm{sec}$ and equation (34) one finds

$$
\left(f_{\pi}\right)_{\text {exp }}=198 \mathrm{MeV} \text {. }
$$

The agreement of equation (40) and (41) is unexpectedly good.

As has been emphasized earlier by one of us (P.G.0.F.) [14], in the context of $S U(6)$ symmet $f_{\pi}$ also determines the matrix element for the process $\rho^{0} \rightarrow e^{+}+e^{-}$. Let us re-emphasize the essential role of the interpretation of the pion's electric $m . s$. radius as the length determin ing $\left|\Psi^{\prime}(0)\right|^{2}$ in equation (36) for our argument.

Finally the result of this section appears to encourage us in trusting solution (29a-30a) o Section 5 .

The next section will discuss whether our trust is compatible with other experiments.

After this paper was submitted for publication we were informed by W. Thirring and by V.F. Weisskopf that they independently arrived at the results of this section.

\section{Can Quarks have a Small Mass ( $\lesssim 500 \mathrm{MeV})$ ?}

In Section 5, we have shown that our zero range bound state model favors the existence of quarks of mass as low as half the central meson mass. In Section 6 we then saw that for such 10 mass quarks a simple theory of $\pi \rightarrow \mu \nu$ decay can be formulated. This theory was found to agree with experiment. The question now is can we tolerate such light quarks? The first objection on can raise is that, would quarks be so light, they should have been observed in low energy experiments. Thus we have to suggest some mechanism to forbid copious low energy quark production One such argument has been advanced by Freund and Oehme [13]. This argument is the following. From $S U(6)$ symmetry and a meson pole model one can derive the relation

$$
\frac{G_{M}^{Q}\left(q^{2}\right)}{G_{E}^{Q}\left(q^{2}\right)}=\mu_{Q}
$$

between the Sachs magnetic and electric form factors of the quark ( $\mu_{Q}=$ total quark magnetic moment in quark magnetons). Now it is well known that the Pauli form factor $F_{2} Q$ of the quark is

$$
F_{2}^{Q}\left(q^{2}\right)=\frac{G_{M}^{Q}\left(q^{2}\right)-G_{E}^{Q}\left(q^{2}\right)}{1-q^{2} / 4 M^{2}} .
$$

By equation (43) $F_{2}^{Q}\left(q^{2}\right)$ would have a pole at $q^{2}=4 M^{2}$ unless the numerator vanishes in this point. Since by (42) the numerator of (43) will not in general vanish at $q^{2}=4 M^{2}$ we have to 
covide $G_{M}^{Q}$ and $G_{E}^{Q}$ with an extra threshold factor $b\left(q^{2}, \delta\right)=\left(1-q^{2} / 4 M^{2}\right)^{\delta}$ with $\delta \geqslant 1$ in order 0 avoid the appearance of the unphysical pole in $F_{2}^{Q}\left(q^{2}\right)$. In the context of the pole model this actor would have to originate in the meson-quark vertex. This means that in our whole disussion in Section 2 we have to replace $g_{i X}^{2} / 4 \pi$ by $g_{i X}^{2} / 4 \pi \cdot b\left(q^{2}, \delta\right)$. It is clear that our conlusions about the $U(6) \times U(6) \times O(3)$ invariance of the zero range approximation remain unhanged, as only ratios of coupling constants (equations (21)) are relevant for this feature.

For $\delta \geqslant 1$ the numerical solutions (29) of the equations (12) are changed and for each inrease of $\delta$ by one unit new solutions appear. It is however remarkable that the solution (29a$0 a$ ) is highly stable against this change. Thus with $\delta \geqslant 1$ (e.g. $\delta \equiv 2$ ), we still find a soluion with $\alpha_{0} \sim 1$. Thus the factor $b\left(q^{2}, \delta\right)$ supresses quark production near threshold. The factor $\left(q^{2}, \delta\right)$ can of course be interpreted as a barrier factor that contains the quarks to within a adius $\tau \sim 1 / m$ within the meson in spite of the small binding [15].

of course our model knows only about the $q \bar{q}$-system nearly at rest in its center of mass frame. owever the introduction of the barrier factor $b\left(q^{2}, \delta\right)$ has asymptotically to be compensated by factor say $\left(q^{2} / \lambda^{2}\right)-\beta$ with $\beta>\delta$ so as to result in an overall asymptotic limit zero for the eson-quark vertex [16]. For $\beta \gg \delta$ quark production will be suppressed at large energies as ell. In practice only very large values of $\beta$ and $\delta$ will do [17].

of course all experimental limits on the quark mass are milder for integrally charged as pposed to fractionally charged quarks. It should be noted that all our arguments are independnt on the charge or statistics of quarks.

Our arguments in this section do by no means prove that quarks have a small mass. They only Indicate the absence of any convincing arguments against such a possibility.

We know that the zero range approximation is still reasonable for large values of the quark mass $M$ in the scalar case [2]. In the light of our previous results it would then be very hard to understand how the known strength of the universal strong interactions could be compatible with a large quark mass.

\section{Conclusions}

We have investigated in this paper a model of mesons as quark-antiquark bound states. Our model, based on a $S U(3)$ invariant zero range approximation, satisfied Lorentz-invariance and elastic unitarity. It led us naturally to meson-quark interactions invariant under the group $M(12) \times O(3)$. Thus $U(6)$ invariance emerged as a dynamical consequence of our scheme. Beyond identifying the large symmetry of our result we were able to calculate the universal strong coupling constants in terms of the ratio of quark to meson masses. From the known meson masses and universal strong coupling constants we arrived at the very low value for the quark mass $\approx 1 / 2 \times$ (meson mass). This permitted us to calculate the $\pi \rightarrow \mu \nu$ decay rate much like one calculates the $2 \gamma$ decay of parapositronium. The result we obtained for the pion lifetime agrees remarkably well with experiment. We finally pointed out that there is no conclusive experimental evidence against such light quarks.

It appears that our results in as much as they extend beyond symmetry considerations do essentially depend on a quark substructure of hadrons. It is therefore an important problem to see whether alternative quark models can reproduce our results. 


\section{References}

1. L. D. LandaU, Sov. Phys. JETP 12, 1294 (1961).

For the case of the deuteron this method has been first implemented in the context of a dispersion theoretic treatment of nucleon-nucleon scattering by $M$. L. GOLDBERGER, Y. NAMBU and R. OEHME, Ann. Phys. 2, 726 (1957). See also V. GRIBOV, B. PERELOMOV and Ya.B. ZELDOVICH, Sov. Phys. JETP 13, 836 (1961).

2. W. THIRRING, Nuovo Cimento 23, 1064 (1962).

3. M. L. GOLDBERger, M. T. GRISARV, S. W. MACDOWELL and D.Y. WONG, Phys. Rev. 120, 2250 (1960).

4. At the same time we have corrected a misprint in the reference [3] where the right-hand sides of equation (4.25d) and (4.25e) are to be multiplied each by an overall factor $(2 J+1)$.

5. R. GATto, L. MAIANI and G. PREPARAtA, Nuovo Cimento 39, 1192 (1965).

6. P.G.0. FREUND, Phys. Rev. Letters 16, 291 (1966).

7. P.G.0. FREUND, Phys. Letters 15, 352 (1965); the numerical factor on the right-hand side of the first equation in the second column of page 352 and in equation (1) of this ref: should read $3 / 5$ instead of $5 / 3$.

8. It is amusing to note that a relation of the type (29a) has been derived from scalar meson dominance of the matrix elements of the trace of the energy momentum tensor using equations (19) in reference [6]. In our model this result is a consequence of bound state dynamics. It thus appears that universality is implemented in our bound state model.

9. K. BAUMANN, P.G.0. FREUND and W. THIRRING, Nuovo Cimento 18, 906 (1960).

10. Actually this turns out not be be necessary. All that matters in this section is that quarki have an effective mass of $\sim m / 2$ within the meson.

11. A. I. AKHIEZER and V.B. BERESTETSKY, Quantum Electrodynamics, p. 349. AEC-tr-2876 (Pt. II).

12. That this is the right procedure can be most easily seen in the context of a Bethe-Salpeter model. There the $B-S$ wave function is

$$
x_{\alpha}^{\beta}(x, y)=\left\langle 0\left|T \psi_{\alpha}(x) \bar{\psi}^{\beta}(y)\right| \pi>\right.
$$

where $\Psi_{\alpha}$ is the 12-component quark Dirac spinor. $f_{\pi}$ is then related to the trace $\left(\gamma_{\mu} \gamma_{5} \lambda_{i}\right)_{\beta}^{\alpha} x_{\alpha}^{\beta}(0,0)$ since the $\psi^{\prime}$ 's are bare fields. No renormalization coefficient and of course no meson pole term appears in the expression of $f_{\pi}$.

13. P.G.0. FREUND and R. OEHME, Phys. Rev. Letters 14, 1085 (1965).

14. P.G.0. FREUND, Nuovo Cimento (in press).

15. A similar proposal has recently been made by M. GELL-MANN, private communication.

16. M. LEHMANN, K. SYMANZIK and W. ZIMMERMANN, Nuovo Cimento 2, 425 (1955).

17. A. MAHESHWARI and E. SCHONBERG, to be published. 